\title{
Is External Ventricular Drainage Life-Saving in Cerebellar Hemorrhages?
}

\author{
Suat Erol Çelik, Ajlan Uzunkol, Buse Sarıgül, Halil Olgun Peker, Yunus Kurtuluş \\ Department of Neurosurgery, Okmeydanı Training and Research Hospital, Istanbul, Turkey \\ Email: suaterolcelik@yahoo.com, ajlanuzunkol@gmail.com, busesarigul90@hotmail.com, \\ halilolgunpeker@gmail.com,ynsmr275@gmail.com
}

How to cite this paper: Çelik, S.E., Uzunkol, A., Sarıül, B., Peker, H.O. and Kurtuluş, Y. (2019) Is External Ventricular Drainage Life-Saving in Cerebellar Hemorrhages? Open Journal of Modern Neurosurgery, 9, 154-163.

https://doi.org/10.4236/ojmn.2019.92015

Received: February 21, 2019

Accepted: April 15, 2019

Published: April 18, 2019

Copyright $\odot 2019$ by author(s) and Scientific Research Publishing Inc. This work is licensed under the Creative Commons Attribution International License (CC BY 4.0).

http://creativecommons.org/licenses/by/4.0/

\begin{abstract}
Background: The treatment of cerebellar hemorrhage $(\mathrm{CH})$ may be different surgery or conservative according to the hematoma volume, compression of vital structures or hydrocephalus existence. In the present study, the authors investigated the risk factors, the indications and the situation of external ventricular drainage (EVD) on the treatment line. Methods: 63 pure cerebellar hemorrhage patients were enrolled in the study. 36 cases underwent surgery; the other 27 were received conservative treatment. 15 and 13 cases received EVD in both groups. Hospital stay and mortality rates were investigated. Results: 4 cases in the conservative group underwent surgery secondary to treatment failure. Both of the groups had equal rates of morbidity and mortality. On the other hand, the group that received surgical intervention had shorter median hospital stay. The EVD does not seem to be life-saving at first but it gives time for preparing for surgery. Conclusions: We found that $\mathrm{CH}$ was strongly associated with early hydrocephalus and mortality. The early diagnosis and surgical evacuation of the mass are mandatory and life-saving if hematoma is larger than $10 \mathrm{ml}$. The EVD may not being a life-saving instrument but majorly it may be a time earning device if acute hydrocephalus present.
\end{abstract}

\section{Keywords}

Cerebellar Hemorrhage, External Ventricular Drainage, Hydrocephalus, Hypertension

\section{Introduction}

Cerebellar hemorrhage $(\mathrm{CH})$ is a sudden life-threating condition that requires quick diagnosis and fast abolishment of therapeutic approaches. Sir Charles Balance performed the first successful surgical removal of hematoma in 1906 [1]. 
Following his first initiative efforts, the cumulative data on the diagnosis, therapeutic approaches and surgery of the cerebellar hemorrhage were increased up to date with enhanced clinical experience.

After 1970s, a new revolution was initiated on neurologic sciences with the invention of the computerized tomography (CT). In the CT era, the time of diagnosis was shortened and wrong diagnoses such as ischemic events were easily eradicated. Moreover; with detailed imaging advances via CT scan, new diagnostic parameters arose such as compression of the fourth ventricle, hydrocephalic changes or hematoma volume in posterior fossa content. These measures facilitated the practitioners to decide better on how to treat the patient either surgically or conservatively.

In the previous literature, there are several studies comparing the outcomes of conservative and surgical management of $\mathrm{CH}$. In the guidelines for the management of spontaneous $\mathrm{CH}$, Broderic et al. suggested surgical evacuation of hematoma, if the diameter is more than $3 \mathrm{~cm}$, if there is neurological deterioration or the patient has brainstem compression and hydrocephalus [2] [3] [4] [5]. A collective database has not been constituted until now; current studies principally consist of uncontrolled, single institution retrospective case series.

Donaeur et al. also suggested the benefits of surgical management. After analysis on previous 21 papers published, they conducted the treatment of $33 \mathrm{pa}-$ tients. They established a management protocol: Group I, small hematoma in good clinical condition treated conservatively; Group II, cases with hydrocephalus treated with EVD; Group III, large hematoma cases treated surgically. Their overall mortality rate was $33 \%$ and the worst prognosis related to patients with an additional intra-ventricular hematoma and men aged between 50 - 70 years with an admission GCS of less than 7 and occluded cisterns [6] [7] [8].

To the author's opinion, the management of patients in good neurologic condition or deep coma is not more complicated in initial surgery or not surgery options. However, the condition is getting complicated after 48 hours from the ictus even in the intensive care unit. The majority of the patients may deteriorate by time caused by ongoing perilesional edema or compression of vital structures such as fourth ventricle, basal cisterns and brainstem. The hematoma volume may also increase with time either via re-bleeding or hyperosmolar effect of clot formation. All of these conditions may show the importance of surgical intervention.

In the present study the authors attempted to try to identify the surgical indications in $\mathrm{CH}$ and detailed the usage of EVD as a time earning device in the setting of such cases managed both surgical and conservatively.

\section{Material and Methods}

There are 63 consecutive patients enrolled in the study between January 2012 and May 2018. Cases of secondary hemorrhage resulting from a cerebral aneurysm rupture, brain tumor bleeding, arteriovenous malformations rupture, 
moyamoya disease or hemorrhagic transformation of infarct area were excluded. All patients had spontaneous pure cerebellar hematoma without structural or vascular abnormality. All patient and family consents with ethical clearance of the study were provided.

Patient characteristics and demographic data are demonstrated in Table 1.

At initial work-up the patient's hematoma volume was calculated into the program of Infinitt PACS system (Seoul, South Chorea). Patient's fourth ventricle configuration was graded via a modified Kirollos system [9]. Grade I: normal size and configuration. Grade II: slightly compressed. Grade III: severely compressed but still have visibility. Grade IV: totally occluded or absent. The condition and compression of the basal cisterns were evaluated according to Jacobs Taneda's criterias [10]. Cisterns were scored by visual inspection using a three-point scale. 1): normal appearance, 2): compressed, indicated unilateral narrowing of a cistern (a slit-like appearance), but CSF still is visible, 3): absent.

According to patient's initial GCS score, hematoma volume and CT-MRI data an algorithm was used for initial management (Figure 1). Moreover this algorithm was used for patients allocation in groups surgical and conservative.

36 of the patient received emergency posterior fossa decompression with hematoma evacuation. Briefly, patients with more than $10 \mathrm{ml}$ hematoma volume and/or GCS score less than 13 had undergone surgery. Surgery was performed in prone position under the cranial fixation with Mayfield three-pin head holder. Extended suboccipital craniectomy with removal of $\mathrm{C} 1$ arc was performed. Right occipital burr-hole was drilled for future CSF diversion. After dural opening, hematoma removal and bleeding control were performed by microsurgery. Dura mater was closed watertight. Postoperative early ( 2 - 3 hours) and late (in 24 hours and day after) CT scans were used for follow-up (Figure 2 and Figure 3 ).

The other 27 patients were treated in a conservative manner. Bed rest, analgesic therapy and sedation were used for initial conservative management (Figure 2). Mannitol and diuretics were added to the therapy when it had been necessary. The 13 cases of the conservative group and 6 cases of the surgery group received EVD in $3-5^{\text {th }}$ days just after acute hydrocephalus has developed.

4 patients of the 13 who received conservative treatment and EVD placement were operated for hematoma evacuation 2 - 4 days interval after initial EVD insertion.

Table 1. Patient characteristics and demographic data.

\begin{tabular}{ccc}
\hline Groups & Surgery (n: 36) & Conservative (n: 27) \\
\hline Age (y) & $66 \pm 17$ & $63 \pm 14$ \\
Sex (F/M) & $15 / 21$ & $11 / 16$ \\
EVD placement (n/\%) & $6(16 \%)$ & $13(48 \%)$ \\
Reoperation & 1 & 4 \\
Hosp/ICU Time (day) & $22 \pm 13 / 12 \pm 6$ & $53 \pm 11 / 41 \pm 9$ \\
Mortality & $6(16.6 \%)$ & $5(18.5 \%)$ \\
\hline
\end{tabular}




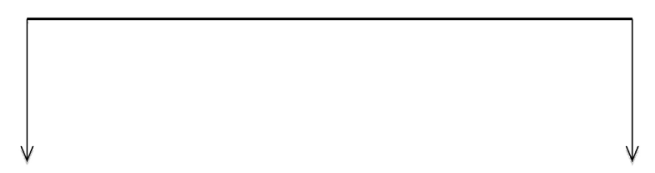

Less than $10 \mathrm{ml}$

More than $10 \mathrm{ml}$

$4^{\text {th }}$ ventricle and cisterns

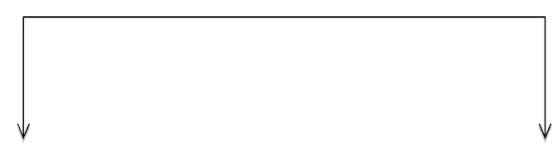

I and II / C1, C2

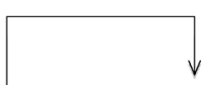

III and V or C3

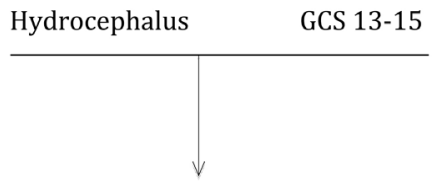

EVD placement

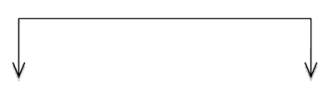

GCS $\leq 12$

Conservative

Surgery

Figure 1. Protocol scheme for the treatment of cerebellar hematomas according to their initial clinical status with neuro-radiological workup.

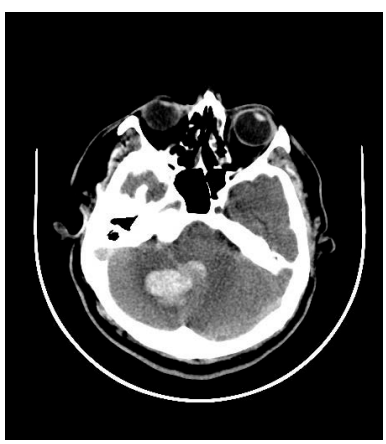

(a)

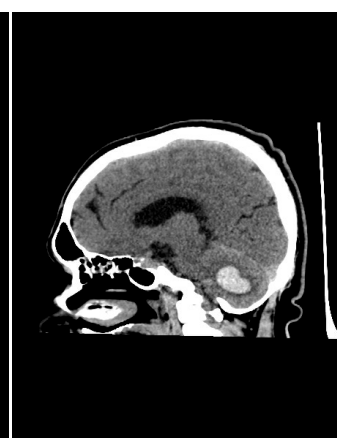

(b)

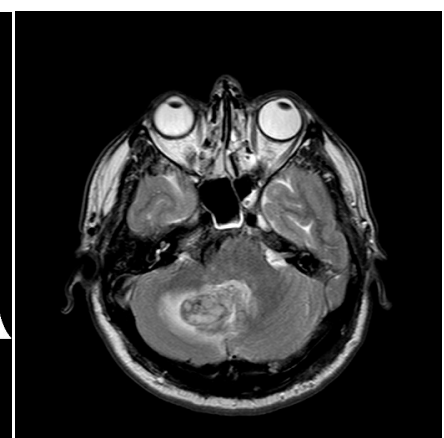

(c)

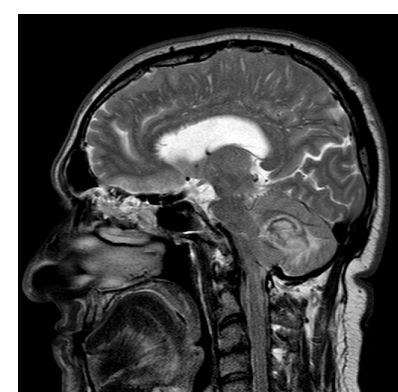

(d)

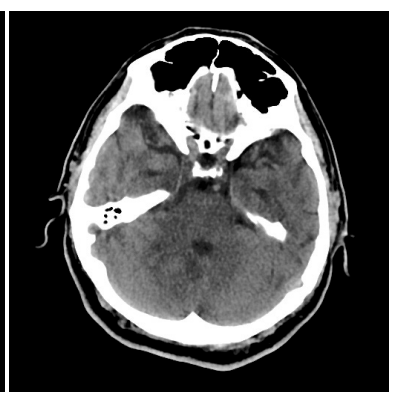

(e)

Figure 2. A 55 years old man admitted to emergency service with hypertensive attack and severe headache. The initial CT scans show ((a) and (b)) $\mathrm{CH}$ with slight $4^{\text {th }}$ ventricle compression and hematoma partly drained into the ventricle. T1 (c) and T2 (d) weighted MRI scans show similar findings. The patient treated conservatively, the CT scan after the 4 weeks show total resolution of $\mathrm{CH}(\mathrm{e})$. 


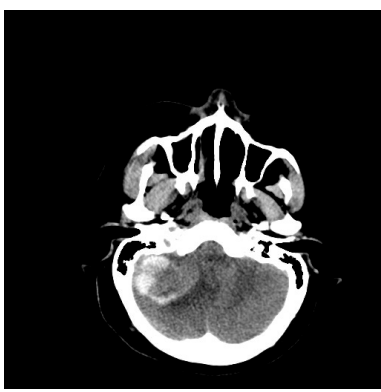

(a)

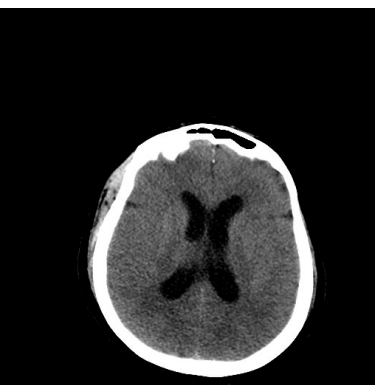

(b)

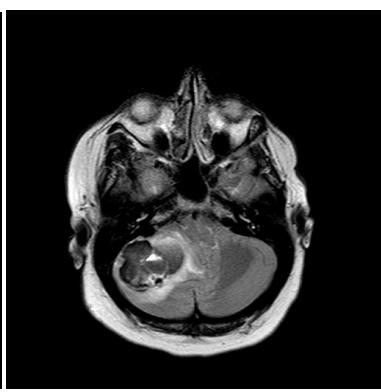

(c)

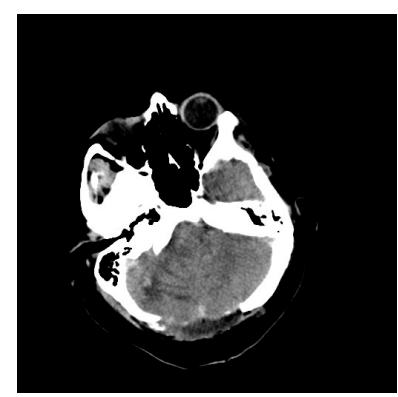

(d)

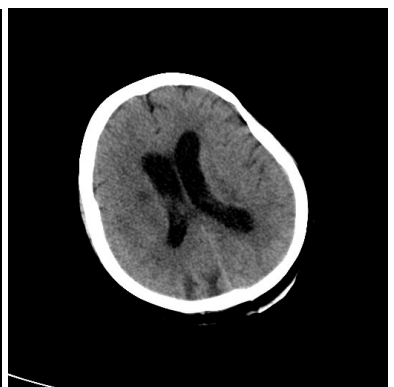

(e)

Figure 3. A 34-year-old women presented sudden deterioration to the emergency service. The initial CT scans ((a) and (b)) show right $\mathrm{CH}$ with compression of the $4^{\text {th }}$ ventricle with no acute hydrocephalus. T1 weighted MRI scan (c) shows hematoma with severe edema and ventricle/cistern compression. The patient underwent emergency decompression and hematoma removal. Postoperative $3^{\text {th }}$ week CT slices ((d) and (e)) show total disappearance of $\mathrm{CH}$ with normal ventricle size.

\section{Results}

Table 1 and Table 2 summarize the results of the patient data. Table 2 also shows CT finding of patients on initial admission. Both of the groups had equal rates of morbidity and mortality. On the other hand, group that received surgical intervention had shorter median hospital stay. The EVD does not seem to be life-saving at first but it gives time for preparing for surgery. 3 patients who could not undergo posterior fossa surgery because of with highly co-morbid status, benefited from EVD placement.

\section{Discussion}

Cerebellar hematoma is mostly seen in middle age or older patients with a peak in fifth decade [11]. Some patients with spontaneous $\mathrm{CH}$ are under warfarin therapy with increased INR ratios plus co-morbid diseases. Most of the patients on warfarin or ASA therapy both have cardiac and neurological ischemic events in their background clinical data. Therefore, they need to be under careful titration of their blood viscosity, platelet and hemoglobin levels prior to surgery. Moreover, such older age group patients may need elongated postoperative ICU treatment with long term hospital stay plus much more physical rehabilitation treatment. Most common cause of spontaneous $\mathrm{CH}$ is hypertension via rupture of microaneurysms in small penetrating vessels. Amyloid angiopathy or 
Table 2. CT characteristics and volume correlation in different grades of $4^{\text {th }}$ ventricle and cistern compression.

\begin{tabular}{cccccc}
\hline $\begin{array}{c}\text { Patient } \\
\text { number }\end{array}$ & $\begin{array}{c}\text { Hematoma } \\
\text { Diam }(\mathrm{cm})\end{array}$ & Volume (ml) & 4. Vent & Cistern & Hyd. \\
\hline 25 & $2-3$ & $7.4 \pm 3.1$ & I & 1 & - \\
13 & $3-4$ & $11.6 \pm 2.3$ & II & $1-2$ & \pm \\
17 & $4-5$ & $22.1 \pm 3.4$ & II-IV & $2-3$ & + \\
8 & $5 \uparrow$ & $34.2 \uparrow$ & IV & $2-3$ & + \\
\hline
\end{tabular}

hemorrhagic transformation of infarct may also be the other reasons [11].

The management of $\mathrm{CH}$ requires early diagnosis of the insult since the pathology is fatal if early surgical intervention is not performed. On the other hand, patients with $\mathrm{CH}$ require detailed preoperative medical evaluation prior to such an extensive surgery.

Many treatment protocols are suggested for $\mathrm{CH}$ although there is no certain consensus on the definite treatment of choice. Removals of hematoma via suboccipital craniotomy, endoscopic evacuation via burr-hole, EVD or conservative management are the options [12].

After their initial diagnosis and prior to surgery, the determination of the treatment type is essential due to above-mentioned reasons. On the initial CT, the diameter and volume of the hematoma, compression of the $4^{\text {th }}$ ventricle or brainstem, presence of hydrocephalus are the main points that need to be enlightened. Salazar is the first author to show the importance of the hematoma size [13]. In a study of 15 patients of $\mathrm{CH}$, he showed that size larger than 3 compounds in 11 cases $(73 \%)$, In large hematoma patients compression of $4^{\text {th }}$ ventricle (80\%), basal cisterns $(60 \%)$ and hydrocephalus (53\%) are correlated with increased mortality. Danouer; in his series of 33 patients with $\mathrm{CH}$ [3], showed the increasing morbidity and mortality with obliteration of perimesencephalic cisterns. He also showed that patients with hemorrhage into vermis or adjacent to $4^{\text {th }}$ ventricle had significantly worse prognosis. These initial studies mainly focused on the hematoma size and settlement of hematoma in the posterior fossa.

Besides evaluating all prognostic indicators; Kiyoyoshi studied 31 patients with spontaneous $\mathrm{CH}$ who had a low GCS at admission and underwent MRI. Obliteration of the $4^{\text {th }}$ ventricle and perimesencephalic cistern and the presence of hydrocephalus in initial tomographic scans and the presence of areas of high signal intensity in the brainstem in T2 weighted images were shown. He suggested that brainstem damage with high signal intensity was a significant prognostic factor for determining outcomes of poor grade $\mathrm{CH}$ [14]. This was the first study to show brainstem ischemia caused by direct compression by hematoma or edema formation independent of other factors. Brain stem function may be evaluated by checking auditory brain stem responses but since urgent intervention is needed, there may be no time for these tests preoperatively [15]. Similar- 
ly, such brainstem ischemia may occur after insertion of EVD only without the evacuation of $\mathrm{CH}$.

Loons et al. suggested a classification for appearance of quadrigeminal cistern in a report involving 49cases on the CT scans. According to the classification; grade I (normal cistern), grade II (compressed cistern), grade III (absent cistern); the size of the hematoma was not relevant to the degree of cisternal compression and the influence of edema and hydrocephalus were more important in overall mass effect. As a result of the study, patients with grade I had a favorable outcome, grade II patients good/moderate outcome, especially patients grade II plus hydrocephalus need urgent evacuation of $\mathrm{CH}$ with insertion of the EVD. Grade III patients had a poor outcome irrespective of all treatment. Mortality was $50 \%$ in grade III; other $50 \%$ was vegetative state. In the study, the early surgery had more helpful [16].

In the prospect of the previouscriteria's such as; hematoma volume, compression of the $4^{\text {th }}$ ventricle, basal cisterns and brainstem ischemia and tonsillar herniation on initial MRI all have clinical importance on morbidity and mortality. The patients with smaller hemorrhage, and alert without hydrocephalus can be followed conservative manner. If the patient had larger hematoma more than 3 $\mathrm{cm}$ with tonsillar herniation, hydrocephalus, brainstem compression or basal cistern occlusion needs surgical evacuation of hematoma. EVD insertion cannot be replaced with main surgery but it can be a time gaining instrument. However, all this management needs close monitoring of the patients, if deterioration happens on the clinical condition of the patient, the urgent CT with rapid tracking of the pathology is crucial. Although the similar conclusions were highlighted by the other studies, if the patients general condition and the hematological values permit to a major surgery, operation with removal of the mass effect seems shortens patients hospital stay with low morbidity and mortality. In the study of Matthew et al. operated $75 \%$ of $48 \mathrm{CH}$ cases. They showed early surgical management in the $\mathrm{CH}$ patients associated with early presentation of CT signs of brainstem compression and acute hydrocephalus led to a good outcome in most patients. The patients initially treated by EVD but more than half of the patients subsequently required evacuation of the clot, but understandably some patients failed to reverse the deterioration [17]. This non-reversal condition may weigh on the craniectomy can be advance to the first line treatment than EVD such larger hematomas. Hoop et al. suggested that level of consciousness is a better prognostic factor than the diameter of hematoma and surgical decompression is indicated in patients with intact brain stem reflexes and impaired consciousness [18].

Kobayashi divided in two parts of their clinical series of 101 cases of $\mathrm{CH}$. They analyzed first 52 cases as a retrospective manner. After this evaluation, the second part of 49 cases was managed insights of new surgical criteria according to Glascow Coma Scale (GCS). Based on these criteria 1, patients with GCS of 14 or 15 and with a hematoma of less than $40 \mathrm{~mm}$ in maximum diameter are treated conservatively. For the patients with GCS of 13 or less on admission or 
with a hematoma measuring $40 \mathrm{~mm}$ or more, hematoma evacuation with de-compressive craniectomy should be treatment of choice; and 3. For the patients whose brain stem reflexes are entirely lost with flaccid tetraplegia or whose general condition is poor surgical therapy was not intended [19]. Luparello and Canevero from Italy also exhibited the importance the GCS, the placement of hematoma (vermian, paramedian, lateral or hemi cerebellar) and the degree of quadrigeminal cistern involvement on their reply to the Kobayashi's study [20]. However Kobayashi's study is the biggest case series including both prospective and retrospective parts. Their criteria are also logic and easy to use in all centers in the world. Moreover, the Glascow Outcome Score may not be predictive for all $\mathrm{CH}$ cases.

Kirollos et al. developed and prospectively applied a new treatment protocol on their 50 patients with $\mathrm{CH}$ according to the $4^{\text {th }}$ ventricle. They graded the appearance of the $4^{\text {th }}$ ventricle on CT scans. Grade I is normal size and configuration. Grade II is partially compressed of distorted, shifted to contralateral side. Grade III is complete obliterated. The degree of fourth ventricle obliteration was correlated with the size and volume of hematoma and presenting GCS score. In conclusion, they suggested that for conscious patients with grade III fourth ventricle compression should undergo urgent clot evacuation before deterioration. Surgical evacuation of the clot may not be required for large hematomas $(>3 \mathrm{~cm})$ if the fourth ventricle is not obliterated at the level of clot. Grade I and grade II with GCS more than 13 were treated conservatively. Ventricular drainage was applied only if the patient had developed hydrocephalus or GCS less than 13. 15 of 25 patients (60\%) with grade I and II compression did not require surgery later on [11].

This suggestion may change in observation time in every day, if the patient has severe brain stem compression or severe peri-hematomal edema formation after the initial insult. Compression of the fourth ventricle may change with time and sometimes the severe hydrocephalus may accompany grade II compression depending on CSF flow dis-regulation.

Based on cumulative data of the previous studies and our series, $\mathrm{CH}$ is a life-threatening condition once it has been diagnosed. Prevalence rate of $\mathrm{CH}$ is increasing in population especially via aging of the people with increased comorbid factors. Mortality varies between 25\% and 57\%. Postoperative outcome is correlated with preoperative clinical condition [10]. Advances in neuroanesthesiology with neurointensive care made it easy to operate such cases in urgent conditions. To the definitive and short time treatment is only possible with surgical evacuation. If the lesion diameter is more than $3 \mathrm{~cm}$, surgical evacuation of hematoma content is the gold standard. EVD is both life-saving and time-gaining instrument if patients have hydrocephalus but can not be undergone urgent surgery.

\section{Conflicts of Interest}

The authors declare no conflicts of interest regarding the publication of this paper. 


\section{References}

[1] Balance, H.A. (1906) Case of Traumatic Haemorrhage into the Left Lateral Lobe of the Cerebellum, Treated by Operation, with Recovery. Surgery, Gynecology \& Obstetrics, 3, 223-225.

[2] Broderic, J.P., Adams, H.P., Barsan, W., Feinberg, W., Feldmann, E., Grotta, J., et al. (1999) Guidelines for the Management of Spontaneous Intracerebral Hemorrhage. Stroke, 30, 905-915. https://doi.org/10.1161/01.STR.30.4.905

[3] Amar, A.P. (2012) Controversies in the Neurosurgical Management of Cerebellar Hemorrhage and Infarction. Neurosurgical Focus, 32, E1. https://doi.org/10.3171/2012.2.FOCUS11369

[4] Hankey, G.J. and Hon, C. (1997) Surgery for Primary Intracerebral Hemorrhage. Is It Safe and Effective? Stroke, 28, 2126-2132. https://doi.org/10.1161/01.STR.28.11.2126

[5] Auer, L.M., Auer, T. and Sayama, I. (1986) Indications for Surgical Treatment of Cerebellar Haemorrhage and Infarction. Acta Neurochirurgica, 79, 74-79. https://doi.org/10.1007/BF01407448

[6] Donauer, E., Loew, F., Faubert, C., Alesch, F. and Schaan, M. (1994) Prognostic Factors in the Treatment of Cerebellar Haemorrhage. Acta Neurochirurgica, 131, 59-66. https://doi.org/10.1007/BF01401454

[7] Freitas, P.E. and Aquini, M.G. (1987) Spontaneous Intracerebellar Hematoma during Childhood. Neurosurgery, 21,103-105. https://doi.org/10.1227/00006123-198707000-00025

[8] Wakai, S. and Nagai, M. (1989) Histological Verification of Microaneurysms as a Cause of Cerebral Haemorrhage in Surgical Specimens. Journal of Neurology, Neurosurgery, and Psychiatry, 52, 595-599. https://doi.org/10.1136/jnnp.52.5.595

[9] Kirollos, R.W., Tyagi, A.K., Ross, S.A., van Hille, P.T. and Marks, P.V. (2001) Management of Spontaneous Cerebellar Hematomas. A Prospective Treatment Protocol. Neurosurgery, 49, 1378-1387. https://doi.org/10.1097/00006123-200112000-00015

[10] Taneda, M., Hayakawa, T. and Mogami, H. (1987) Primary Cerebellar Hemorrhage: Quedrigeminal Cistern Obliteration on CT Scan as a Predictor of Outcome. Journal of Neurosurgery, 67,545-552. https://doi.org/10.3171/jns.1987.67.4.0545

[11] Datar, S. and Rabinstein, A. (2014) Cerebellar Hemorrhage. Neurologic Clinics, 32, 993-1007. https://doi.org/10.1016/j.ncl.2014.07.006

[12] Hackenberg, K., Unterberg, A., Jung, C.S., Bösel, J., Schönenberger, S. and Zweckberger, K. (2017) Does Suboccipital Decompression and Evacuation of Intraparenchymal Hematoma Improve Neurological Outcome in Patients with Spontaneous Cerebellar Hemorrhage? Clinical Neurology and Neurosurgery, 155, 22-29. https://doi.org/10.1016/j.clineuro.2017.01.019

[13] Salazar, J., Vaquero, J., Martinez, P., Santos, H., Martinez, R. and Bravo, G. (1986) Clinical and CT Scan Assessment of Benign versus Fatal Spontaneous Cerebellar Hematomas. Acta Neurochirurgica, 79, 80-86. https://doi.org/10.1007/BF01407449

[14] Kiyoyuki, Y., Meguro, K., Fujita, K., Narushima, K. and Nose, T. (1999) Postoperative Brainstem High Intensity Is Corraleted with Poor Outcomes for Patients with Spontaneous Cerebellar Hemorrhage. Neurosurgery, 45, 1323-1328. https://doi.org/10.1097/00006123-199912000-00010

[15] Yanaka, K., Meguro, K., Fujita, K., Narushima, K. and Nose, T. (2000) Immediate Surgery Reduces Mortality in Deeply Comatose Patients with Spontaneous Cerebellar Hemorrhage. Neurologia Medico-Chirurgica, 40, 295-300. 
https://doi.org/10.2176/nmc.40.295

[16] Loon, J.V., Calenberg, F.V., Goffin, J. and Plets, C. (1993) Controversies in the Management of Spontaneous Cerebellar Haemorrhage. A Consecutive Series of 49 Cases and Review of the Literature. Acta Neurochirurgica, 122, 187-193. https://doi.org/10.1007/BF01405527

[17] Mathew, P., Teasdale, G., Bannan, A. and Oluoch-Olunya, D. (1995) Neurosurgical Management of Cerebellar Haematoma and Infarct. Journal of Neurology, Neurosurgery, and Psychiatry, 59, 287-292. https://doi.org/10.1136/jnnp.59.3.287

[18] Hoop, V.D., Vermeulen, M. and Van Gijin, J. (1988) Cerebellar Hemorrhage: Diagnosis and Treatment. Surgical Neurology, 29, 6-10. https://doi.org/10.1016/0090-3019(88)90115-2

[19] Kobayashi, S., Sato, A., Kageyama, Y., Nakamura, H., Watanabe, Y. and Yamaura, A. (1994) Treatment of Hypertensive Cerebellar Hemorrhage-Surgical or Conservative Management. Neurosurgery, 34, 246-251. https://doi.org/10.1227/00006123-199402000-00006

[20] Luparello, V. and Canavero, S. (1995) Treatment of Hypertensive Cerebellar Hemorrhage-Surgical or Conservative Management Comment. Neurosurgery, 37, 552-553. https://doi.org/10.1227/00006123-199509000-00037 